\title{
A monitoring system based on phasor measurement units with variable reporting rates
}

\author{
Paolo Castello $^{1}$, Carlo Muscas ${ }^{1}$, Paolo Attilio Pegoraro ${ }^{1}$, Sara Sulis ${ }^{1}$ \\ ${ }^{1}$ Department of Electrical and Electronic Engineering, University of Cagliari, Cagliari, Italy
}

\begin{abstract}
Classical Wide-Area Monitoring Systems (WAMSs) are characterized by a hierarchical architecture, which is composed of Phasor Measurement Units (PMUs) and different levels of Phasor Data Concentrators (PDCs). The WAMS, based on synchrophasor technology, was originally designed for transmission systems; nevertheless, with the development of the smart grid paradigm, the benefits of this technology are being extended to distribution networks. Normally, PMUs send measurement data at a high and constant reporting rate to guarantee the monitoring of dynamic events in an electric transmission network. However, typical communication systems that are expected to be used by distribution system operators are generally shared and/or public. In this case, the bandwidth available among PMUs and PDCs, or among the PDCs and the control center, is strictly dependent on the type of communication channel that is used and on the level of network traffic. In this context, a new transmission logic for the transfer of data between PMUs and PDCs, based on the knowledge of the ongoing conditions of the electric grid, can be implemented. The strategy proposed in this paper is to increase the measurement reporting rate only when the electric system changes from a steady-state condition to a dynamic and potentially unsafe one, without modifying the overall accuracy of the PMU measurement process. The risk of losing important information related to a dynamic event is mitigated by sending to the PDC (after the event detection) measurements relating to the pre-trigger time interval in a burst of data packets that allow insight into the signal evolution.
\end{abstract}

\section{Section: RESEARCH PAPER}

Keywords: PMU; PDC; synchrophasor; cloud; WAMS; variable reporting rate

Citation: Paolo Castello, Carlo Muscas, Paolo Attilio Pegoraro, Sara Sulis, A monitoring system based on phasor measurement units with variable reporting rates, Acta IMEKO, vol. 7, no. 4, article 11, December 2018, identifier: IMEKO-ACTA-07 (2018)-04-11

Editor: Alexandru Salceanu, "Gheorghe Asachi" Technical University of lasi, Romania

Received April 7, 2018; In final form July 3, 2018; Published December 2018

Copyright: (C) 2018 IMEKO. This is an open-access article distributed under the terms of the Creative Commons Attribution 3.0 License, which permits unrestricted use, distribution, and reproduction in any medium, provided the original author and source are credited.

Corresponding author: Paolo Castello, e-mail: paolo.castello@diee.unica.it

\section{INTRODUCTION}

Wide-Area Monitoring Systems (WAMSs) based on synchronized measurements are characterized by a hierarchical architecture composed of Phasor Measurement Units (PMUs) and different levels of Phasor Data Concentrators (PDCs) [1]. The PMU is the "sensor" device that measures electrical quantities, such as voltage and current phasors, frequency, and rate of change of frequency (ROCOF), with an accurate time tag based on Coordinated Universal Time (UTC) and usually obtained from a GPS receiver or through IEEE 1588 (Precision Time Protocol) synchronization.

The role of the PDC is to collect and align measurements provided by the PMUs and to forward this data to the next higher PDC level and, finally, to the control center [2] that is responsible for the evaluation of the overall state of the electric grid. The WAMS, based on synchronized measurements, was originally designed for transmission systems; however, since the introduction of the smart grid framework, the benefits of synchrophasor technology are moving also to distribution networks [3]-[7].

The use of PMUs in the distribution network context represents a new challenge: standalone PMUs and PDCs could be replaced by dedicated functionalities implemented in Intelligent Electronic Devices (IEDs) [8] or by existing measurement devices upgraded in order to build an Internet of Things (IoT) network with synchrophasor functionality [9]. In a synchrophasor system suitable for distribution grids, several measurement devices are necessary, and in this new scenario, a classical hierarchical architecture might be inadequate, as it might be unable to manage many PMUs and/or PMU-enabled instruments. One solution could be to replace the hierarchical structure of PDCs with a less expensive and rapidly scalable structure based on cloud computing technology.

Normally, in a WAMS transmission system, PMUs send data at a constant rate of 50-60 frames per second (fps) to guarantee the monitoring of dynamic events [10]. However, the communication systems used by distribution system operators 


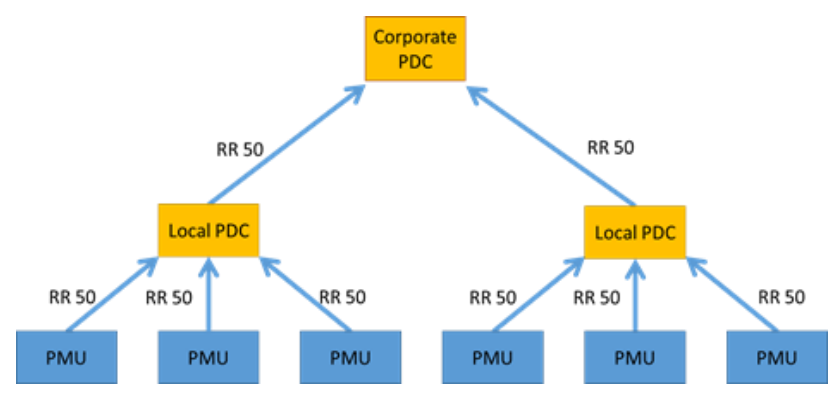

Figure 1. Diagram of the WAMS hierarchy based on PMUs and different levels of PDCs with a fixed and constant reporting rate of $50 \mathrm{fps}$.

(DSOs) are expected to be shared and/or public [11] (alternative solutions based on a private cloud with a private communication channel may be too expensive for DSOs). In this case, the bandwidth available for the devices involved is strictly dependent on the type of communication channel that is adopted [12]

In this context, the present paper proposes a new logic aiming to manage the dynamic change in the reporting rate (RR) of a PMU. The proposal is based on the outcomes of [13], in which the concept of a PMU adapting its RR in order to increase it only when the electric system changes from a steady state to a dynamic and potentially unsafe condition was firstly presented. The PMU is intended to store all the measurement data at the maximum rate inside the PMU and to send, after a change detection, pretrigger information to upper levels.

In this paper, a real architecture that can operate in tandem with commercial devices to manage the above paradigm is introduced. The risk of losing important information concerning the beginning of a dynamic event due to a low steady-state RR is mitigated by sending the information relating to the pre-trigger period to a standard compliant PDC as soon as possible with a suitable policy.

The proposed architecture comprises a real PMU prototype that is able to send data with a variable $R R$ without affecting the accuracy of the measurement process. The PDC responsible for receiving the data is a conventional open-source software PDC with a specific setup that is able to manage the variable data streams from different PMUs. In this way, it is not necessary to implement a specific device to receive variable rates in the measurement architecture, i.e., the PMU prototype can operate in a pre-existing synchrophasor measurement architecture. To the knowledge of the authors, this proposal is the first example of a PMU designed with an adaptive functionality for the transmission of measurement data.

The policies are tested under simulation and the whole architecture is experimentally validated in a laboratory setup.

\section{THE PROPOSED ARCHITECTURE}

Figure 1 shows the typical hierarchical architecture of a WAMS based on the synchrophasor technology described in the IEEE standard C37.118.2-2011 [2], with the PMUs acting as "sensor elements" of the monitored network at the bottom and two levels of PDCs (typically referred to as "local" and "corporate" concentrators) above. In this case, the PMUs send their measurements over the communication network in data packets in compliance with the standard protocol [2] by reporting up to $50 \mathrm{fps}$ for a system frequency of $50 \mathrm{~Hz}$. It is also possible to find commercial PMUs with RRs higher than those required by the standard.

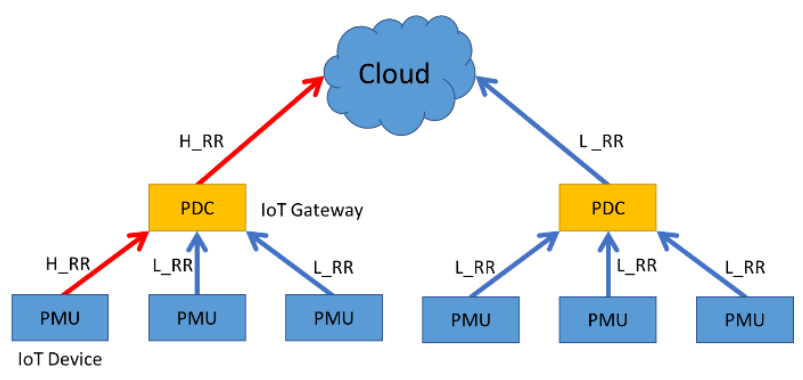

Figure 2. Proposed architecture based on flexible PMUs and a single level of PDCs, exploiting different (high and low) reporting rates.

At each level, the PDC builds a single output data stream from the time-aligned input streams. The synchrophasor data latency at PDC output and, usually, the time for processing incoming data increase in size following each step in the PDC hierarchy.

The number of PMUs required for the electric distribution network is expected to be higher with respect to the transmission grid, due to the large number of nodes that must be monitored. Consequently, the number and size of data streams could become difficult to manage.

Figure 2 illustrates the architecture proposed for a distribution network's synchrophasor system. The communication channel is shared and/or public. In Figure 2, there is a single level of the PDC that acts as a gateway between the devices in the field and the cloud, where the measurements are processed depending on the application (state estimation for real-time control, but also offline applications as post-mortem analytical tools [14]).

The PMU continuously produces and stores the data internally with the maximum RR available (e.g., H_RR = $50 \mathrm{fps}$ ) but, in the presence of a steady-state condition, it sends the data to the PDC with a lower RR (e.g., L_RR = 1 fps).

It is important to recall that in the design of a PMU compliant with [1], the value of RR determines not only the number of measurements per second that is sent to the PDC, but also, for each compliance class and each nominal system frequency, the measurement performance of the device under specific test conditions. Thus, in the proposed architecture, where the actual $\mathrm{RR}$ of the PMU is constant (and the high-rate measurements are continuously stored in a circular buffer of suitable capacity), the metrological characteristics of the device do not change with the output RR, which, on the contrary, can be modified depending on the operating condition of the electric grid.

In the presence of a dynamic event detected in the input signals (e.g., a given threshold is exceeded), the PMU can activate the highest output RR and send the pre-trigger data to the PDC, flushing the buffer [13].

\subsection{Data handling with variable reporting rate}

Devices based on synchrophasor technology must handle measurements at least at the mandatory reporting rates of 10, 25, and $50 \mathrm{fps}$ (for a system frequency of $50 \mathrm{~Hz}$ ). However, other reporting rates are permissible, i.e., lower and higher than the mandatory values. Thus, in this scenario, an RR of $1 \mathrm{fps}$ indicates the lowest rate considered. Figure 3 shows different policies that can be adopted by a PMU to send the measurement data, and an event occurring at $t=1 \mathrm{~s}$.

Case 0 represents the standard policy with a fixed RR. In particular, it represents an $\mathrm{RR}$ equal to $1 \mathrm{fps}$ where every measurement is time tagged at the occurrence of the UTC second. 


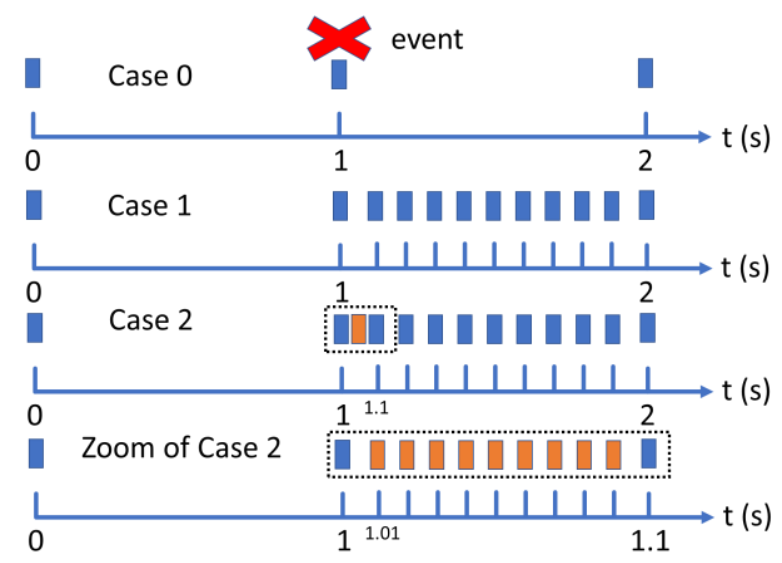

Figure 3. Different measurement reporting policies with a more detailed representation of the case with pre-trigger data.

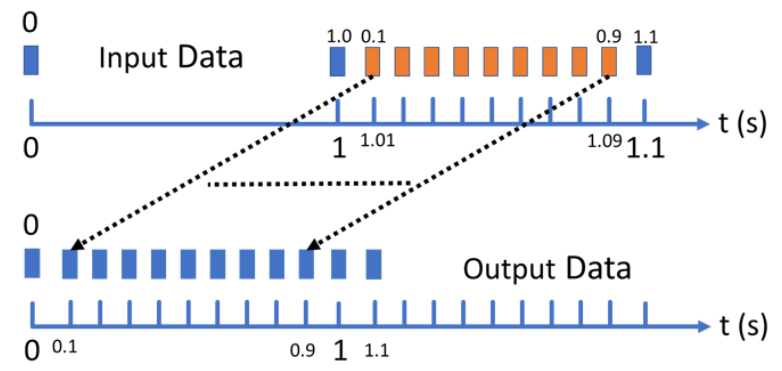

Figure 4. PDC input and output data with the time-aligned operation concerning the pre-trigger data.

Case 1 adopts a dynamic transmission policy based on [9] where, at the occurrence of an event of interest ( $t=1 \mathrm{~s})$, the RR changes from the lowest rate (1 fps between $0 \mathrm{~s}$ and $1 \mathrm{~s}$ ) to a higher rate $(10 \mathrm{fps}$ after $t=1 \mathrm{~s})$. The RR of $10 \mathrm{fps}$ is the lowest $\mathrm{RR}$ subject to the dynamic requirements of the standard [1] and, thus, can be considered as suitable to follow varying electrical signals.

Case 2 shows the policy adopted in this paper to also send the measurement data relating to the pre-event time interval [13]. The PMU sends the data with the lowest RR until a significant variation is detected in the monitored quantities $(t=1 \mathrm{~s})$. After the detection, the PMU starts sending the stored non-real-time measurements relating to the pre-trigger period. The pre-trigger measurements are sent within a burst of data packets (nine packets in this case, corresponding to the nine non-transmitted measurements of the pre-event interval of $1 \mathrm{~s}$ ) at $100 \mathrm{fps}$ (Figure 3 , Case 2: orange blocks between $t=1 \mathrm{~s}$ and $t=1.1 \mathrm{~s}$ ). Figure 3 shows a zoom of Case 2 and represents the details of the transmission of measurements between $1 \mathrm{~s}$ and $1.1 \mathrm{~s}$. After sending such data packets, the PMU returns to the real-time data transmission, but with an increased RR of $10 \mathrm{fps}$, chosen to better follow the detected dynamics of the ongoing event. It is important to highlight that even if the pre-trigger data is sent with a RR of $100 \mathrm{fps}$, the measurements are always relative to a data evaluation every $100 \mathrm{~ms}$. Indeed, the timestamps of the measurements are $100 \mathrm{~ms}$ from each other. This solution is a trade-off between continuously following the signal in real time and recovering pre-trigger data offline. In fact, it allows the PMU to send the pre-event data before the occurrence of a new realtime data packet and before the PDC realigns all the measurement data in a timely manner. Figure 4 shows the time

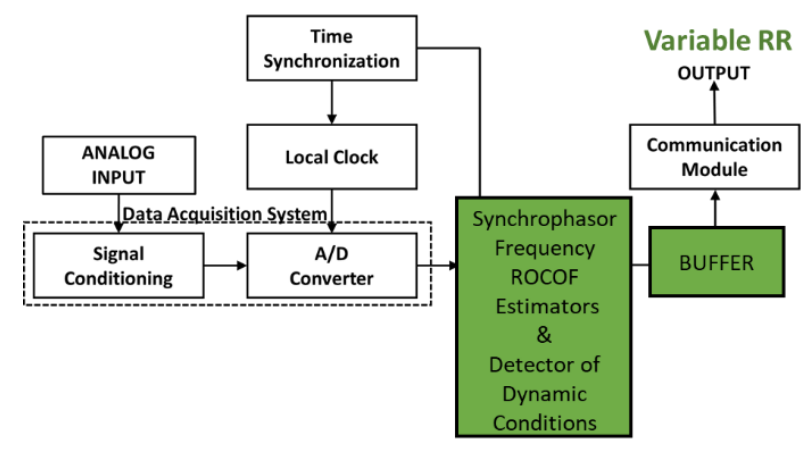

Figure 5. Schematic representation of the PMU prototype.

alignment operation of the PDC responsible for receiving the variable RR. The input data received from the PDC is not timealigned, and the time between the packets is not constant. The PDC of a classic WAMS expects to receive data at regular time intervals, and considering the latency characteristics of the network channel, after a given waiting time, it assumes the missing measurements are lost [15]. For this reason, in the proposed architecture, the waiting time is exploited in order to manage incoming data streams at different reporting rates without any ad hoc implementation.

Equation (1) shows the different latency contributions used to set the correct values of the waiting time:

$$
T_{\text {wait }}=T_{q}+\operatorname{Max} P M U_{L}+T_{N L} \text {. }
$$

The PDC is set to wait the incoming data for a time that is equal to the maximum time allowed in the buffer implemented in the PMU ( $T_{q}$ corresponding to the pre-trigger data window), a time depending on the maximum measurement process of PMUs $\left(\operatorname{Max} P M U_{L}\right)$, and the latency between the PMU prototype and the PDC ( $T_{N L}$, corresponding to network latency). For $\operatorname{Max} P M U_{L}$, the value of the PMU reporting latency suggested in the synchrophasor standard for a RR of $10 \mathrm{fps}$ and for the $\mathrm{M}$ class configuration is considered:

$$
\operatorname{Max} P M U_{L}=7 / R R
$$

In this way, the data for a pre-trigger event is considered simply as time-delayed data packets from a PDC point of view.

It is important to highlight that in order to allow the operation of the PDC, in IEEE standard C37.118.2, a new configuration frame can be sent when a PMU changes the format of the data stream. The RR is specified in the reporting rates information and every change in the parameters of the PMU would require a new configuration frame before the change occurs. In this paper, in order to save the communication bandwidth and always considering the format of data frames as constant, the rate value in the configuration frames is set as the highest used. In this way, even if the actual RR is lower, the PDC considers all the missing data as delayed and as missing (at the end of the waiting time), without any loss of generality.

To implement the variable RR policy, the architecture of a PMU needs to be enhanced with other technical solutions. Figure 5 is a schematic representation of the proposed PMU prototype. The device, which uses the same measurement algorithm as [5], acquires the relevant signals by synchronizing the acquisition process in order to time tag every signal sample. The detection of the dynamic event is implemented as a user-selected threshold depending on the values of interest. In particular, considering the scope of this work, the monitored variable is the frequency deviation from the nominal value. When a dynamic condition is 


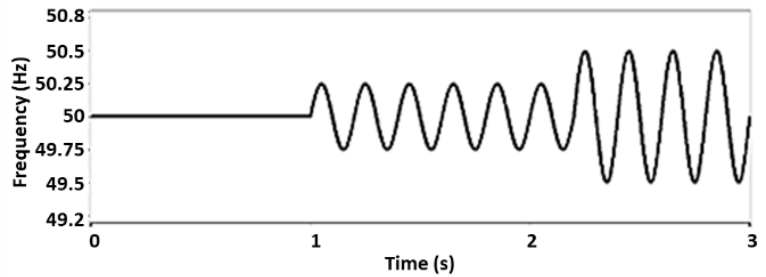

Figure 6. Frequency of the test signal used for validating the proposed method based on the detection of the off-nominal frequency.

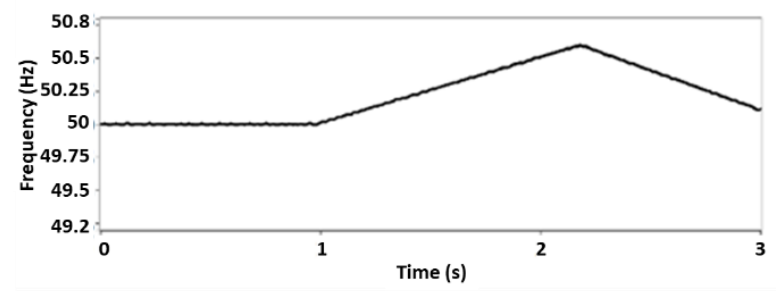

Figure 7. Frequency variation with a positive and negative linear ramp with different values of ROCOF.

detected, the highest output RR is activated, which also maintains high values when the monitored quantity returns under the threshold level for a programmable time so that bounces around the threshold can be avoided. A central element of the architecture is the circular buffer, where the measurements are stored at the maximum internal RR of $50 \mathrm{fps}$, maintaining the accuracy of the measurement, which is characteristic of this RR.

\section{TEST SETUP}

In the following simulation tests and real scenarios obtained by means of software modules implemented in the LabVIEW environment, the performance of the proposed monitoring system in a dynamic event is shown.

As a representative example of possible data management policies, the detection of a significant frequency deviation from the nominal value of $50 \mathrm{~Hz}$ is addressed. To save a significant portion of the monitored phasors, the PMU is equipped with a circular buffer, which can host $1 \mathrm{~s}$ of data.

First, simulation test cases will be reported using RRs of $1 \mathrm{fps}$ and $50 \mathrm{fps}$ for the analysis. Then, in the real scenario, the measurement RRs configured in the prototype for the tests are 1 fps and $10 \mathrm{fps}$, while, as described above, a packet rate of $100 \mathrm{fps}$ can be used in the transmission module.

\subsection{Simulation tests}

Communication between the PMU and the PDC functionality is developed in the LabVIEW environment by means of independent virtual instruments. In the following steps, the pretrigger data is sent in a single data packet following the event detection. This technique requires a specific PDC functionality that is able to manage single data packets with the stored pretrigger information and the associated timestamps. The aim of the following tests is to illustrate the impact of the policies on the event reconstruction.

\subsubsection{Phase modulation test case}

The synchrophasor standard [1] suggests the use of a sinusoidal phase modulation signal to test the bandwidth of a PMU using different modulation frequencies and a modulation level of $k_{a}=0.1 \mathrm{rad}$. In such case, the maximum absolute variation from the nominal system frequency is:

$$
\Delta_{f \max }=k_{a} \cdot f_{m},
$$

where $f_{m}$ is the adopted modulation frequency. The highest $f_{m}$ suggested by the standard is $5 \mathrm{~Hz}$, which corresponds to $\Delta_{f \max }=0.5 \mathrm{~Hz}$. On the contrary, considering a constant $f_{m}$, the deviation from the nominal system frequency is proportional to the modulation level $k_{a}$, and $\Delta_{f \max }$ varies with the modulation level. It is thus possible to impose a threshold for dynamic condition detection on the maximum frequency deviation.

In the simulation tests, $\Delta_{f}=0.4 \mathrm{~Hz}$ is used to discriminate a strong dynamic condition, and upper and lower frequency thresholds are defined as follows:

$$
f_{\text {threshold }}=f_{o} \pm \Delta_{f} \text {. }
$$

Figure 6 shows the frequency of the adopted test signal as a function of time, where $k_{a}$ changes with time. At the beginning, the test signal is in a steady-state condition at the nominal frequency ( $k_{a}=0$, no modulation).

After one second has elapsed, the test signal is phase modulated with a modulation frequency of $5 \mathrm{~Hz}$ and two different levels of modulation factor. Between $1 \mathrm{~s}$ and $2.2 \mathrm{~s}$, the modulation factor is $k_{a}=0.05 \mathrm{rad}$, which leads to a maximum frequency deviation that is equal to $\Delta_{f \max }=0.25 \mathrm{~Hz}$.

After $2.2 \mathrm{~s}$, a stronger dynamic condition occurs when $k_{a}$ becomes $0.1 \mathrm{rad}$. In this case, the maximum deviation from the nominal system frequency is $\Delta_{\text {fmax }}=0.5 \mathrm{~Hz}$, i.e., above the fixed threshold.

As recalled above, in order to avoid continuous changes between high and low reporting rates, the highest value is maintained for $T_{\text {hold }}$ seconds (10 $\mathrm{s}$ for all the tests) after the monitored parameter returns below the threshold. The aim is to light up the ongoing phenomenon and trigger a more accurate observation of its evolution.

\subsubsection{Frequency ramp test case}

A second simulated scenario is represented by a variation of the frequency, with different values of ROCOF. The frequency trend shown in Figure 7 comprises:

- a steady-state condition at the nominal system frequency between $0 \mathrm{~s}$ and $1 \mathrm{~s}$,

- a positive frequency ramp at a rate of $0.5 \mathrm{~Hz} / \mathrm{s}$ from $1 \mathrm{~s}$ to $2.2 \mathrm{~s}$, and

- a frequency ramp with negative ROCOF (-0.6 $\mathrm{Hz} / \mathrm{s}$ ) from $2.2 \mathrm{~s}$ to the end of the simulation.

During these events, the values of the frequency are explained as:

$$
f(t)=f_{\text {start }}+R_{f} \cdot\left(t-t_{\text {start }}\right),
$$

where $f_{\text {start }}$ and $R_{f}$ are the signal frequency at the ramp starting time $t_{\text {start }}$ and the frequency ramp ROCOF, respectively.

To simulate a real signal acquired from the power system, a uniform white noise with a SNR level of $50 \mathrm{~dB}$ is added to the test signal.

Using the same threshold value applied in the dynamic conditions of the phase modulation test, the signal reaches the threshold level after $t=1.78 \mathrm{~s}$. This will be discussed in detail in the next section. 


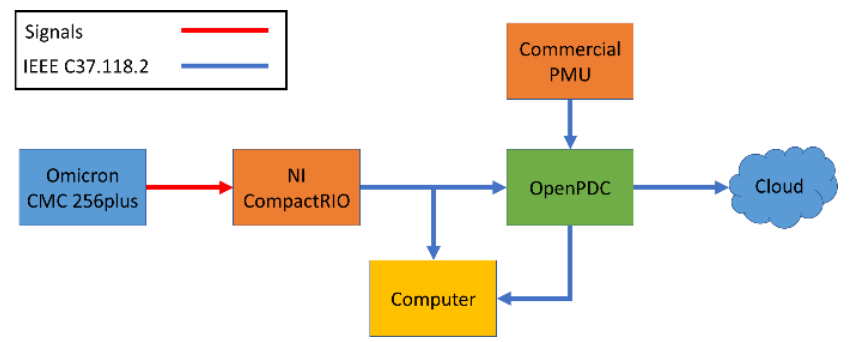

Figure 8. Representation of the test setup for the real scenario.

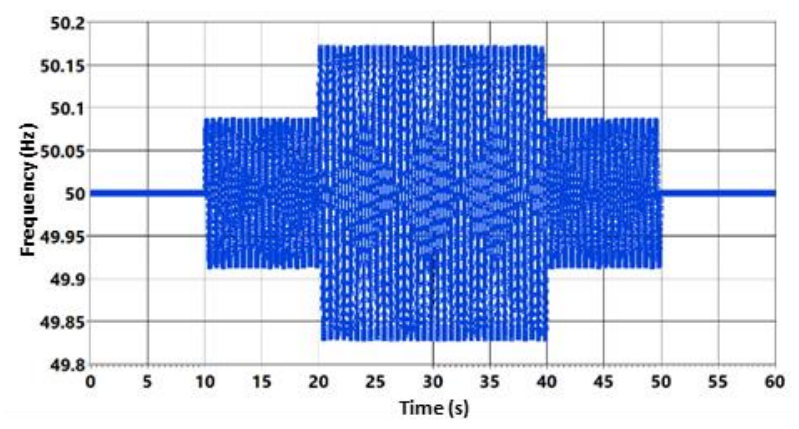

Figure 9. One minute of the frequency trend of the test signal produced by the signal generator.

\subsection{Architecture of the real test scenario}

Figure 8 illustrates the test setup that is used to verify the feasibility of the proposed approach in a real context. The calibrator Omicron CMC 256plus provides accurate voltage signals of amplitude equal to $10 \mathrm{~V}$. The generator is programmed to reproduce an event represented by a waveform with different levels of phase modulation (different $k_{a}$ ) and a common frequency modulation $f_{m}$ of $1.7 \mathrm{~Hz}$ chosen in order to emphasize the behavior of the monitored frequency (Figure 9). The signal generator is programmed to reproduce the waveform every minute, in a time-synchronized manner, so that the performance of the system prototype can be evaluated continually. The frequency trend shown in Figure 9 comprises:

- a steady-state condition at the nominal system frequency between $0 \mathrm{~s}$ and $10 \mathrm{~s}$ and between $50 \mathrm{~s}$ and $59 \mathrm{~s}$,

- a modulation factor of $k_{a}=0.05 \mathrm{rad}$ from $10 \mathrm{~s}$ to $20 \mathrm{~s}$ and from $40 \mathrm{~s}$ to $50 \mathrm{~s}$, and

- a modulation factor of $k_{a}=0.1 \mathrm{rad}$ from $10 \mathrm{~s}$ to $20 \mathrm{~s}$ and from $40 \mathrm{~s}$ to $50 \mathrm{~s}$.

The signal generator provides the test signal to the prototype implemented in a Compact Reconfigurable Input and Output (CompactRIO) device manufactured by National Instruments, which adopts a real-time controller and a Field Programmable Gate Array (FPGA). The CompactRIO is composed of three main modules, used to implement the prototype:

1. The I/O modules provide the signals of interest for the overall system. In this prototype, the NI-9215 data acquisition module, a 16 -bit, $\pm 10 \mathrm{~V}, 100 \mathrm{kSa} / \mathrm{s}$ simultaneous channel, is used to acquire the voltage from the signal generator. Moreover, a GPS receiver that is often used in time-keeping applications due to its availability, low price, and high accuracy [16] is considered. The NI-9467 synchronization module is used to receive the accurate time reference provided by the UTC to synchronize the overall system, with a time accuracy of $\pm 100 \mathrm{~ns}$.

2. The I/O FPGA, in this case a NI 9113, is configured to manage the data acquired from the I/O modules and to maintain the relationship with the acquired signals and the absolute time reference.

3. The real-time controller, in this case a NI 9024, is responsible for computing the values of interest, verifying the level of the monitored quantities, storing the data in the buffer, and managing communication.

The PMU prototype sends the data to a workstation with PDC functionality provided with the software OpenPDC from the Grid Protection Alliance [17]. The OpenPDC software can manage the data provided by PMUs in the field, and it is used in different scientific applications to enhance proposed functionalities, thanks to its open-source characteristic [18]-[20].

In this work, the OpenPDC receives data from two PMUs: the proposed prototype with the variable RR and a commercial PMU set to the fixed RR of 1 fps. The commercial PMU is not connected to a specific signal source, because its role is only to send the synchronized data (which is continuously measured) and to test the correct process of the PDC, in terms of data collection and time alignment in the presence of an incoming fixed RR in addition to the variable RR stream.

To maintain the accuracy of the data, the PDC is set to wait $T_{\text {wait }}=2 \mathrm{~s}$, considering the maximum dimension of the internal buffer of the PMU prototype $\left(T_{q}=1 s\right)$, the PMU latency for the PMU configuration that is being tested $\left(\operatorname{Max} P M U_{L}=0.7 \mathrm{~s}\right)$, and a reasonable value of network latency $\left(T_{N L}=0.3 \mathrm{~s}\right)$. In this way, the PDC can align the pretrigger data concerning the detected event correctly. This solution increases the overall communication latency of the system because the PDC waits for two seconds before forwarding the data to the cloud. Nevertheless, this solution also allows for the use of the proposed transmission strategy in a preexiting measurement system. It is important to recall that the aim of this proposal is to save communication bandwidth by reducing, when unnecessary, the amount of data that is sent to the control center. In the proposed architecture, it is also possible to set the specific waiting time on a per-PDC basis, depending on the area that is being monitored and on the available devices.

\section{TEST RESULTS}

\subsection{Simulation results}

\subsubsection{Phase modulation test case}

Figure 10, Figure 11, and Figure 12 plot the frequency measurements received by the PDC relating to the test signals described in the previous section.

The three different cases corresponding to the three different RR policies illustrated above are:

- $\quad$ Case 0: the frequency measurements sent by the PMU with a constant $\mathrm{RR}=1 \mathrm{fps}$, thus giving four measurements.

- Case 1: the frequency measurements sent with a step change in the RR around the event detection without pre-trigger data transmission (similar to [9]).

- Case 2: frequency measurements received by the PDC when the $1 \mathrm{~s}$ pre-trigger data is sent along with the variable RR. 


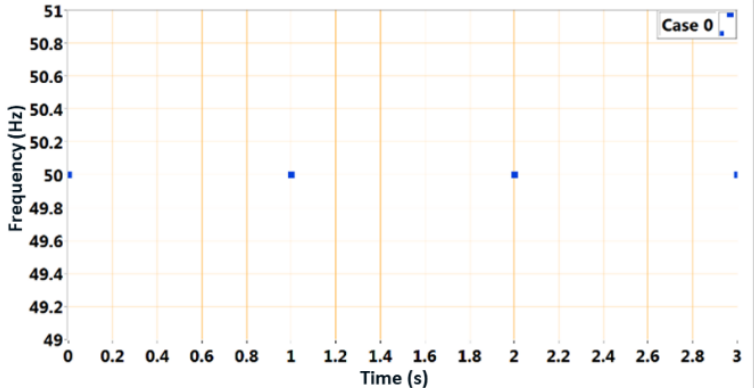

Figure 10. Case 0: data received from the PDC with a constant RR policy equa to $1 \mathrm{fps}$.

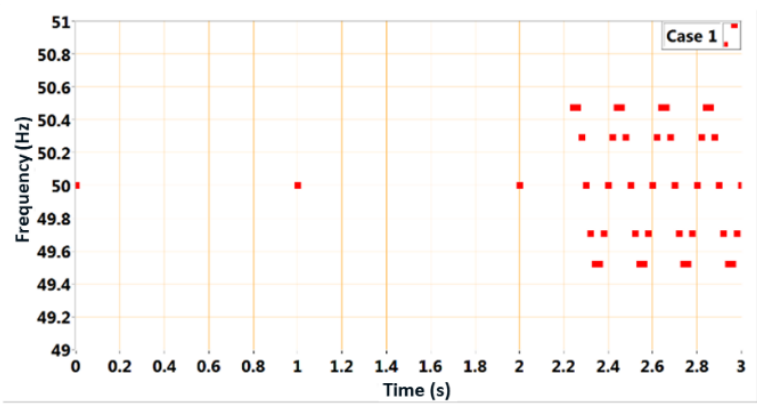

Figure 11. Case 1: data received from the PDC with a variable RR policy.

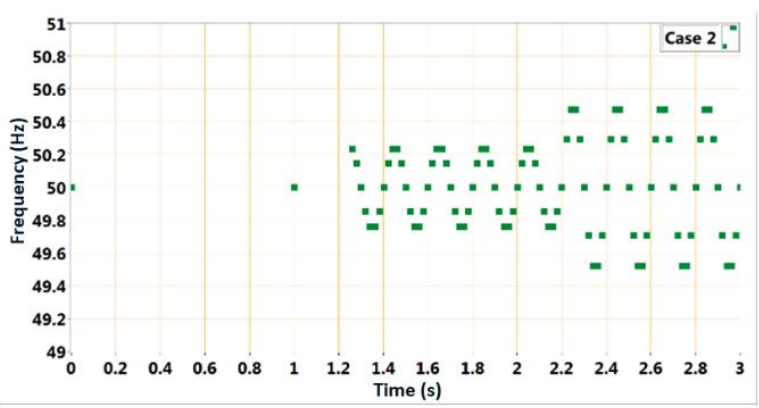

Figure 12. Case 2: data realigned by the PDC with the variable RR and pretrigger data.

In Case 0 , the measurements are received every second, and as also explained in the synchrophasor standard, the RR is insufficient in terms of its ability to follow the sinusoidal variation of the frequency (it is far below the Nyquist frequency of the occurring dynamics).

Case 1 represents an adaptive RR, and the highest RR (50 fps) starts when the value of the frequency exceeds the imposed threshold $(t=2.22 \mathrm{~s})$. In this case, it is possible to follow the dynamic event as it occurs, but it is not possible to understand how the frequency evolved to such a critical level that distinguishes the steady state from the dynamic condition.

Case 2 covers this gap, as the measurements relating to the pre-trigger period are sent to the PDC in the first packet following the event. The PDC aligns the data to show the evolution of the frequency before the event occurred.

\subsubsection{Frequency ramp test case}

The second simulation test scenario presents a steady-state signal at the system frequency combined with two linear frequency ramps with different levels of ROCOF, as described in Section 3.1.2.
Figure 13, Figure 14, and Figure 15 show the measurement data received by the PDC from the PMU in accordance with the aforementioned three different RR policies.

Figure 13 represents the frequency monitored for $4 \mathrm{~s}$, with the lowest RR considered (Case 0). It is important to highlight that for RR lower than $10 \mathrm{fps}$, PMUs are not subject to the dynamic requirements. Therefore, their configuration and measurement algorithm can be very different from those that are suitable for following the signal changes, as is the case in linear frequency ramp conditions.

The trend of the frequency measurements received by the PDC from a PMU with a variable RR (Case 1) is shown in Figure 14. To save communication bandwidth, the PMU can adapt between two RRs: $1 \mathrm{fps}$ when the frequency is under the threshold and $50 \mathrm{fps}$ after the threshold has been exceeded $(t=$ $1.78 \mathrm{~s})$.

The policy of a variable RR highlights the dynamic event and saves the communication bandwidth in the synchrophasor system during the steady-state period, but it does not allow the whole trend of the event to be followed before the threshold level is reached.

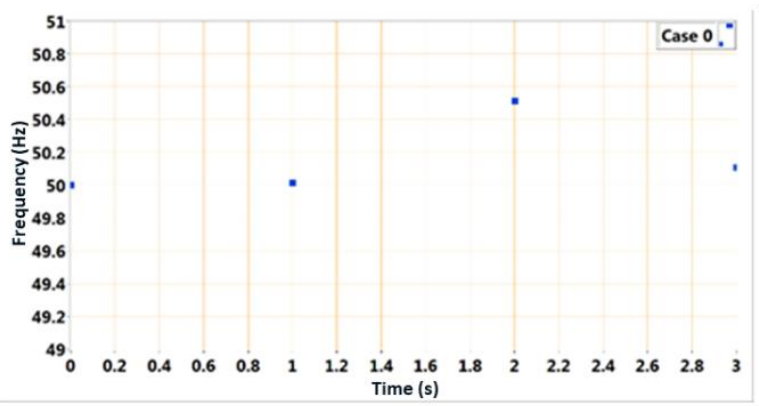

Figure 13. Case 0: data received from the PDC with a constant RR policy equal to $1 \mathrm{fps}$.

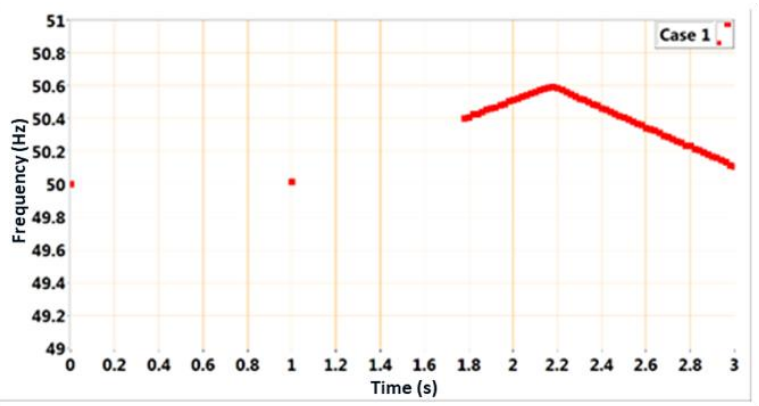

Figure 14. Case 1: data received from the PDC with a variable RR policy.

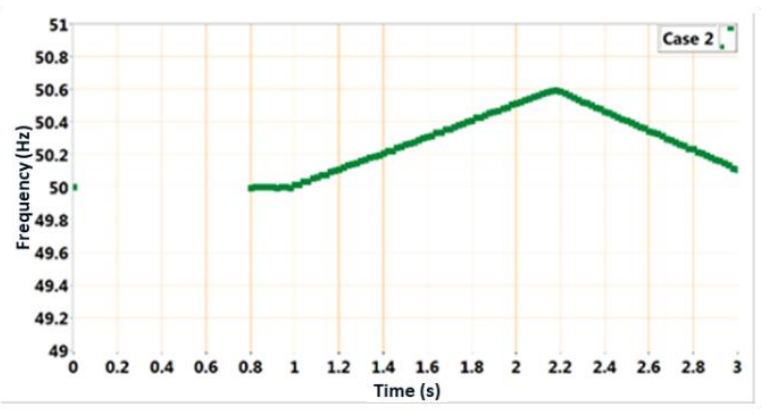

Figure 15. Case 2: data realigned by the PDC in case of variable RR and pretrigger data. 
To overcome this issue, a pre-trigger policy should be applied. Figure 15 shows the data that is received and aligned by the PDC from the PMU with variable RRs and pre-trigger data. In this case, it is possible to follow the trend of the signal whose frequency has exceeded the threshold. The data stored in the circular buffer covers $1 \mathrm{~s}$ at $\mathrm{RR}=50 \mathrm{fps}$ before the trigger event, which causes the highest RR to be activated $(t=0.8 \mathrm{~s})$. Such a buffer size allows a relevant retrospective interval while avoiding a backup packet transmission that is too large.

Moreover, it is interesting that in both Case 1 and Case 2, during the negative ramp period, the dynamics are followed also below the threshold limit, thanks to the holding period introduced to avoid the jerky evolution of the RR.

\subsection{Real scenario results}

The tests on the real scenario focus on the capability of the monitoring architecture to handle the variable $\mathrm{RR}$ and the retrospective data provided by the PMU prototype. The PDC collects and aligns the input data from the two PMUs with different rates without receiving the configuration frames before the rate variation. This behavior is permitted by the PDC, considering all the data as delayed or missing depending on the elapsed time.

Figure 16 shows the graphic interface of OpenPDC Manager, which was used to configure the input and output data streams managing options. The figure shows the synchronized data streams of the PMU prototype (PMU VR, variable rate) and of the commercial PMU (PMU B). In Figure 16, the beginning and end of the frequency evaluation corresponding to the oneminute input signal loop are visible.

The output data of the PDC is shown in Figure 17, Figure 18, and Figure 19, which corresponds to the data collected from the workstation running the PDC software.

Figure 17 shows the PDC output data relating to the received stream of the PMU prototype for a complete loop of the test signal. The monitoring activity can be divided into two categories: the first, from $0 \mathrm{~s}$ to $19 \mathrm{~s}$ and from $48 \mathrm{~s}$ to $59 \mathrm{~s}$, is characterized by a low monitoring rate, while the second, from $19 \mathrm{~s}$ to $48 \mathrm{~s}$, is characterized by a higher reporting rate.

In Figure 18, the data handling operation of the PDC is represented in more detail. The frequency value highlighted by the red circle is the first frequency value overcoming a threshold limit of $50.15 \mathrm{~Hz}\left(\Delta_{f}=0.15 \mathrm{~Hz}\right)$, taken from [21] as a value used as trigger condition for the monitoring of frequency events. The PDC output appears to change the RR from $1 \mathrm{fps}$ to $10 \mathrm{fps}$ before the detection instant, in the highlighted area between $19 \mathrm{~s}$ and $20 \mathrm{~s}$. This behavior is the consequence of the data alignment operation of the PDC, also performed on the pre-trigger measurements considering the correct time stamps.

Finally, Figure 19 reports the frequency of the generated signal loop, monitored from $35 \mathrm{~s}$ to $59 \mathrm{~s}$. The figure shows the change of rate, from $10 \mathrm{fps}$ to $1 \mathrm{fps}$, ten seconds after the actual detection of the last frequency value over the imposed threshold, as the result of the hold-on policy. The rate of $10 \mathrm{fps}$ allows for the decrease in the deviation from the nominal frequency up to the extinguishment of the electrical phenomenon to be followed.

\section{CONCLUSION}

To bring the benefits of synchronized measurements from the transmission to the distribution level, the classic architecture of the wide area monitoring system needs to be adapted to the new context. In this proposal, a new data handling method for a synchrophasor system based on variable RR and pre-trigger data transmission has been illustrated using simulation and real test cases. To efficiently manage data transmission and to save bandwidth in the case of shared and/or public communication channels, the RR of the PMU is changed using a selectable threshold, driven by the dynamic events that are possible in the

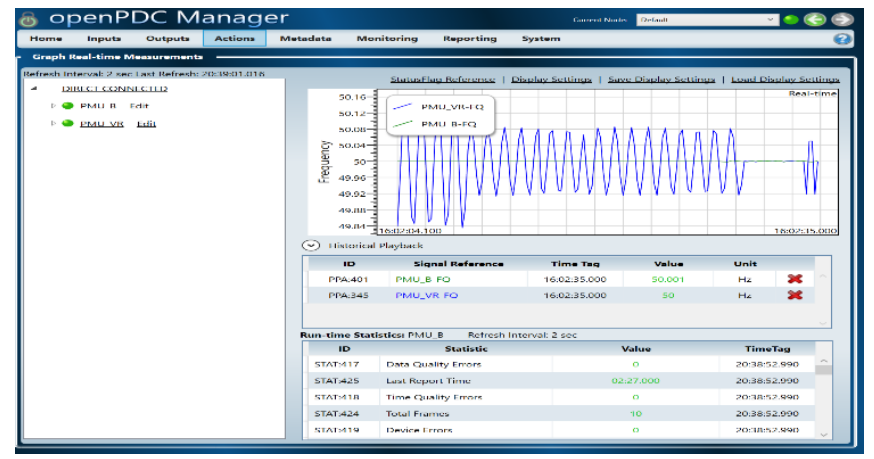

Figure 16. The graphic interface of Open PDC manager: PMU prototype (PMU $\mathrm{VR}$ ) and commercial PMU (PMU B) are listed.

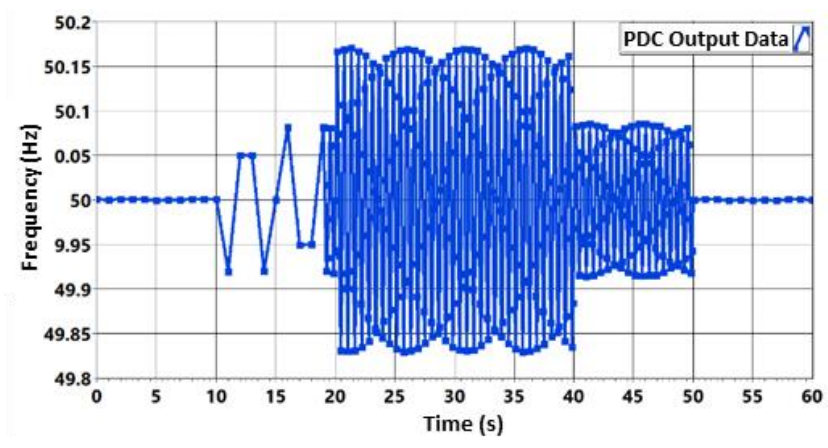

Figure 17. Data realigned by the PDC in the case of variable RR and pre-trigger data.

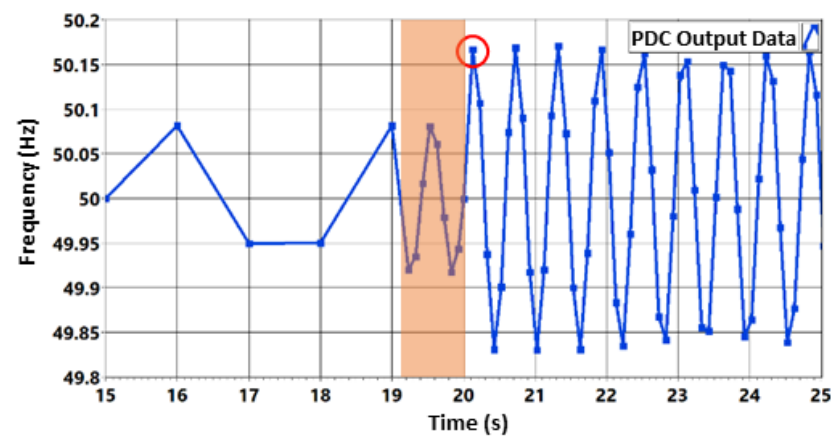

Figure 18. Data realigned by the PDC in the case of change of reporting rates and pre-trigger data.

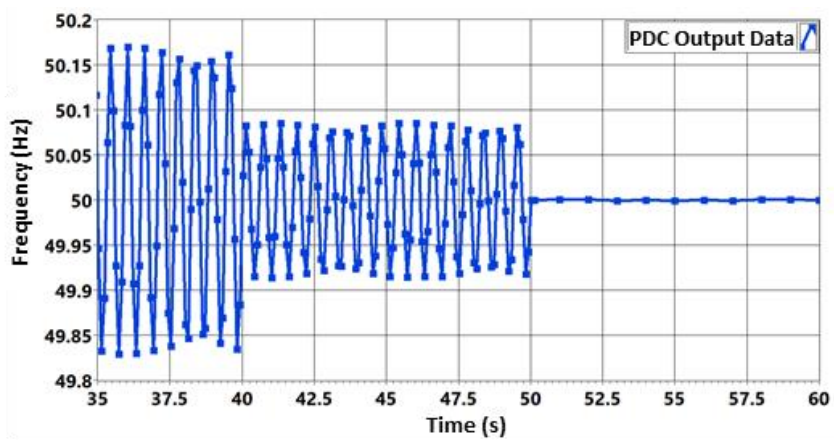

Figure 19. Detailed trend of data realigned by the PDC relating to the last part of the monitored frequency. 
electric distribution systems. The possibility of losing significant information about the beginning of a dynamic event is mitigated by sending the measurements relating to the pre-trigger interval to the PDC so that the PDC can realign them and report a useful measurement context for different applications. Examples of the application of the proposed solution have been discussed, thus proving how the use of such adaptive policies can help save bandwidth while preserving important information when dynamic events occur. Policies that can follow both very fast events and slower evolutions have been defined. The pre-trigger data is always useful to complete the available information, because it is possible to analyze the event origin or early behavior without the risks of oversensitivity and thus being affected by negligible fluctuations. The pre-existent measurement systems, including commercial PMUs, can easily adopt the proposed solution; thus, variable RRs can also be managed in the presence of hybrid infrastructures.

\section{ACKNOWLEDGEMENT}

The research activities described in this paper have been conducted in the context of the research and development project 'Cagliari2020', partially funded by the Italian University and Research Ministry (grant\# MIUR_PON04a2_00381).

\section{REFERENCES}

[1] IEEE, "IEEE Standard for Synchrophasor Measurements for Power Systems”, IEEE Std C37.118.1-2011 Revis. IEEE Std C37118-2005, Dec. 2011, pp. 1-61.

[2] IEEE, "IEEE Standard for Synchrophasor Data Transfer for Power Systems", IEEE Std C37.118.2-2011 Revis. IEEE Std C37118-2005, Dec. 2011, pp. 1-53.

[3] D. Bulic, R. Jancic, V. Kirincic, S. Skok, "Monitoring of low voltage facilities using synchronized phasor measurements", Proc. of 36th International Convention on Information Communication Technology Electronics Microelectronics (MIPRO), May 20-24, 2013, Opatija, Croatia, pp. 1231-1234.

[4] M. Pignati, M. Popovic, S. Barreto, R. Cherkaoui, G. Dario Flores, J. Le Boudec, M. Mohiuddin, M. Paolone, P. Romano, S. Sarri, T. Tesfay, D. Tomozei, L. Zanni, "Real-time state estimation of the EPFL-campus medium-voltage grid by using PMUs", Proc. of the IEEE PES Innovative Smart Grid Technologies Conference (ISGT), Feb. 18-20, 2015 Washington, DC, USA.

[5] P. Castello, J. Liu, C. Muscas, P. A. Pegoraro, F. Ponci, A. Monti, "A fast and accurate PMU algorithm for "P+M" class measurement of synchrophasor and frequency", IEEE Trans. Instrum. Meas. 63(12) (2014) pp. 2837-2845.

[6] A. von Meier, D. Culler, A. McEachern, R. Arghandeh, "Microsynchrophasors for distribution systems", Proc. of the IEEE PES Innovative Smart Grid Technologies Conference (ISGT), Feb. 1922, 2014, Washington, DC, USA.

[7] P. Tosato, D. Macii, D. Brunelli, "Implementation of phasor measurement units on low-cost embedded platforms: A feasibility study", Proc. of the IEEE International Instrumentation and
Measurement Technology Conference (I2MTC), May 22-25, 2017, Turin, Italy.

[8] P. Castello, P. Ferrari, A. Flammini, C. Muscas, P. A. Pegoraro, S. Rinaldi, "A distributed PMU for electrical substations with wireless redundant process bus", IEEE Trans. Instrum. Meas. 64(5) (2015) pp. 1149-1157.

[9] P. A. Pegoraro, A. Meloni, L. Atzori, P. Castello, S. Sulis, "PMUbased distribution system state estimation with adaptive accuracy exploiting local decision metrics and IoT paradigm", IEEE Trans. Instrum. Meas. 66(4) (2017) pp. 704-714.

[10] NERC, "Reliability Guideline, PMU Placement and Installation, December 2016", [Online] Available: https://www.nerc.com/comm/PC Reliability Guidelines DL/ Reliability \%20Guideline \%20-\%20PMU \%20Placement.pdf [Access date 02.01.2019].

[11] C-DAX Deliverable D5.2, "Test Results of Field Trial with CDAX Compatible Grid Devices" [Online] Available: https://cordis.europa.eu/project/rcn/106390/factsheet/en [Access date 02.01.2019].

[12] V.C. Gungor, D. Sahin, T. Kocak, S. Ergut, C. Buccella, C. Cecati, G.P. Hancke, "Smart grid technologies: communication technologies and standards", IEEE Transactions on Industrial Informatics 7(4) (2011) pp. 529-539.

[13] P. Castello, C. Muscas, P. A. Pegoraro, S. Sulis, "New Approach for Measurement Data Handling in Cloud-Based Synchrophasor Systems for Smart Grids", Proc. of IMEKO TC4 Symposium, Sept. 14-15, 2017, Iasi, Romania, pp. 320-324.

[14] J. De La Ree, V. Centeno, J. S. Thorp, A. G. Phadke, "Synchronized phasor measurement applications in power systems", IEEE Transactions on Smart Grid, 1(1) (2010) pp. 2027.

[15] IEEE, "IEEE Guide for Phasor Data Concentrator Requirements for Power System Protection, Control, and Monitoring", IEEE Std C37.244-2013, May 2013, pp. 1-65.

[16] V. R. Ogrizovic, J. Marendic, S. Renovica, J. Gucevic, "Testing GPS generated 1PPS against a rubidium standard", ACTA IMEKO 2(1) (2013) pp. 7-11.

[17] Grid Protection Alliance, "OpenPDC" [Online] Available: https://github.com/GridProtectionAlliance/openPDC [Access Date 02.01.2019].

[18] N. Gellerman, P. Ranganathan, R. Vallakati, A. Mukherjee, "User interface for situational awareness of openPDC", Proc. of the North American Power Symposium (NAPS), Pullman, WA, U.S.A., Sept. 7-9, 2014.

[19] D. Anderson, C. Zhao, C. Hauser, V. Venkatasubramanian, D. Bakken, A. Bose, "Intelligent Design: Real-Time Simulation for Smart Grid Control and Communications Design", IEEE Power and Energy Magazine, 10(1) (2012) pp. 49-57.

[20] M. Golshani, G. A. Taylor, I. Pisica, P. Ashton, "Laboratory-based deployment and investigation of PMU and OpenPDC capabilities", Proc. 10th IET International Conference on AC and DC Power Transmission (ACDC 2012), Dec. 4-5, 2012, Birmingham, UK.

[21] Z. W. Li, O. Samuelsson, R. Garcia-Valle, "Frequency deviations and generation scheduling in the nordic system", Proc. of IEEE Trondheim PowerTech, June 19-23, 2011, Trondheim, Norway. 